\section{YW2-01 IMMORTALIZED NEURAL STEM CELLS PROMOTE A RECOVERY OF VOIDING FUNCTION IN SPINAL CORD INJURED RATS}

\section{Department of Urology, Hokkaido University Graduate School of Medicine}

Takahiko Mitsui

Cellular transplantation into injured spinal cord promotes both axonal regrowth and functional recovery. We investigated whether immortalized neural stem cells (EG6 cells), transplanted into the injured spinal cord of the rat, would produce recovery of lower urinary tract function. Spinal cords of female Wistar rats were injured by compression. BrdU labeled EG6 cells were transplanted into the injured spinal cord 9 days postoperatively. Operatedcontrol rats received intraspinal injections of culture medium. EG6 cells, identified with BrdU immunocytochemistry, survived in the injured spinal cord on day 28. Voided volume per micturition in a metabolic cage increased in $\mathrm{EG}$ 6-transplanted rats on day 28 after transplantation $(\mathrm{P}<0.05)$, but not on day 14. Micturition pressure in cystometry on day 28 decreased in $\mathrm{EG} 6$ transplanted rats compared with controls $(\mathrm{P}<0.005)$. Although voided volume was not different, postvoid residual was less in EG6-transplanted rats than controls $(P<0.05)$. Thus, voiding efficiency was greater in EG6transplanted rats than controls $(\mathrm{P}<0.05)$. Transplanted EG6 cells thus survive in the injured spinal cord and promote recovery of voiding function. Neural stem cells transplantation is a promising modality for the treatment of lower urinary tract dysfunction following spinal cord injury.

\section{YW2-02 IMMUNONEUTRALIZATION OF NERVE GROWTH FACTOR (NGF) IN LUMBOSACRAL SPINAL CORD RE- DUCES DETRUSOR OVERACTIVITY IN SPINAL CORD INJURED RATS}

\footnotetext{
Department of Urology, Shinshu University

Satoshi Seki

We investigated the effects of intrathecal application of NGF antibodies (NGF-

$\mathrm{Ab}$ ) on detrusor overactivity (DOA) in chronic spinalized (SCI) rats. In adult female rats, an intrathecal catheter was implanted at the level of the L6-S1 spinal cord, followed by complete transection of Th8-9 spinal cord. Ten days after spinalization, the intrathecal catheter was connected to an osmotic pump for continuous delivery of either vehicle or NGF-Ab for 2 weeks. In awake cystometry the number of non-voiding contractions (NVC), maximal pressure of NVC and maximal micturition pressure were significantly decreased in NGF-Ab-treated, compared with vehicle-treated SCI rats. In ELISA the NGF levels in the bladder, $\mathrm{L} 6$ spinal cord and L5-S1 DRG of vehicle-treated SCI rats was 1.6-4.8 times higher than in spinal intact rats. After NGF-Ab treatment NGF levels were significantly lower in L6-S1 DRG (30-35\%) and the L6 spinal cord $(53 \%)$ in comparison to levels in vehicle-treated SCI rats.

Increased levels of NGF in the bladder, spinal cord and DRG were associated with DOA after SCI. Immunoneutralization of NGF in the spinal cord suppressed NGF levels in L6-S1 DRG, which contain bladder afferent neurons, and also suppressed DOA. Thus suppression of NGF levels in afferent pathways could be useful for treating DOA associated with SCI.
}

\section{YW2-03 Bladder dysfunction caused by} ischemia-reperfusion

\author{
Department of Surgery, Division of Urology, Tottori \\ University Faculty of Medicine \\ Motoaki Saito
}

We attempted to investigate the bladder function in acute urinary retention and subsequent catheterization in the rat bladder. Our data suggest that acute urinary retention induces ischemia and subsequent catheterization induces reperfusion in the bladder. Moreover, bladder dysfunction following of catheterization is caused by free radicals, and oxygendelivered free

radicals play an important role in the bladder. Since NO is well known as free radical species, we evaluated the effect of NO and its inhibitors on ischemia-reperfusion injury in the rat bladder utilizing pharmacological and pathological methods. 1) Rat bladder injuries were dependent on the duration of ischemia :2) Ischemia-reperfusion caused infiltration of leukocytes and ruptures of microcirculations in the submucosa and smooth muscles without corresponding sloughing of mucosal cells in the rat bladder ;3) Ischemia induced by clamping of the rat abdominal aorta caused reduction in contractile responses to carbachol of the bladder dome, and subsequent reperfusion caused additional damage to smooth muscle judged by functional study ; 4) the peak ratio of apoptosis in the bladder is reached 3 days after the insult of 30 minutes of ischemia, an effect that can be significantly prevented by treatment with L-NAME. 5) Ischemiareperfusion injury was prevented by treatment with $\mathrm{NO}$ inhibitors.

\section{YW2-04 Testosterone can be a therapeutic agent for LUTS}

\section{Department of Urology, Teikyo University school of medicine}

Satoru Muto

Introduction: We already reported that neuronal nitric oxide synthase deficient $(\mathrm{KO})$ mice had reduced $\mathrm{NO}$ concentrations at the bladder and urethra, and these mice showed frequent urination. We have used these $\mathrm{KO}$ mice successfully as an animal model for so-called lower urinary tract symptom (LUTS). Using this model we examined therapeutic effects of testosterone for LUTS.

Materials and Methods: We compared urination patterns and urinary NOx secretion between male wild-type (WT) and KO mice with or without testosterone treatment.

Results : Compared with WT mice, KO mice showed a greater frequency of urination during 24-hour observation period (4.2 vs 6.3 times/day, $\mathrm{P}=0.006$ ) without difference in total voided volume. While testosterone treatment did not affect urination patterns in WT mice, it decreased frequency of urination (6.3 vs 4.1, $\mathrm{P}=$ $0.0018)$ and increased voided volume $(0.19 \mathrm{vs} 0.30 \mathrm{ml}, \mathrm{P}=0.023)$ in KO mice. No significant changes of urinary NOx secretion were noticed before and after administration of testosterone in WT and KO mice.

Conclusions : Testosterone treatment can be a therapeutic option for LUTS. 